\title{
Dynamic subscales in the finite element approximation of thermally coupled incompressible flows
}

\author{
Ramon Codina* ${ }^{* \dagger}$ and Javier Principe \\ Universitat Politècnica de Catalunya, Jordi Girona 1-3, Edifici C1, Barcelona 08034, Spain
}

\begin{abstract}
SUMMARY
In this paper, we propose a variational multiscale finite-element approximation for the incompressible Navier-Stokes equations using the Boussinesq approximation to model thermal coupling. The main feature of the formulation in contrast to other stabilized methods is that we consider the subscales as transient. They are solution of a differential equation in time that needs to be integrated. Likewise, we keep the effect of the subscales both in the nonlinear convective terms of the momentum and temperature equations and, if required, in the thermal coupling term of the momentum equation. Apart from presenting the main properties of the formulation, we also discuss some computational aspects such as the linearization strategy or the way to integrate in time the equation for the subscales. Copyright (c) 2007 John Wiley \& Sons, Ltd.
\end{abstract}

Received 5 December 2006; Revised 25 January 2007; Accepted 1 February 2007

KEY WORDS: thermally coupled flows; dynamic subgrid scales; stabilized finite-element methods

\section{INTRODUCTION}

Thermally coupled incompressible flows are of particular interest from the numerical point of view for different reasons. Apart from their obvious practical interest, very often these flows exhibit instabilities and even transition to turbulence in situations that, in spite of their complexity, are easier to analyse than for isothermal flows. The numerical modelling of these instabilities that take place in rather simple cases is an excellent test for numerical formulations.

In this paper, we propose a finite-element formulation for thermally coupled flows based on the variational multiscale formalism [1]. The basic idea is to split the unknowns, velocity, pressure and

\footnotetext{
*Correspondence to: Ramon Codina, Universitat Politècnica de Catalunya, Jordi Girona 1-3, Edifici C1, Barcelona 08034, Spain.

${ }^{\dagger}$ E-mail: ramon.codina@upc.edu

Contract/grant sponsor: Spanish Ministerio de Ciencia y Tecnología; contract/grant number: VEM2003-20069-C03-03 Contract/grant sponsor: Generalitat de Catalunya (Catalan Government)

Contract/grant sponsor: European Social Fund
} 
temperature, into their finite-element component and a subgrid scale component, hereafter referred to as subscale. The particular approximation used for these subscales defines the numerical model. The main feature of the model we propose is that we consider the subscales time dependent and that we keep their effect in all the terms of the equations to be solved, both the nonlinear convective terms of the momentum and the heat equation and in the coupling term due to the Boussinesq model.

The basic formulation for isothermal incompressible flows is described in [2]. As it is explained there, considering the subscales time dependent and tracking them along the iterative process to deal with the nonlinear terms has several benefits, such as a better performance in time of the final formulation, the conservation of momentum or the possibility to model turbulence. In this paper, we extend the formulation to thermally coupled flows using the Boussinesq approximation and discuss some implementation aspects of the formulation not touched in [2].

The need to stabilize the standard Galerkin finite-element approximation comes from two main sources, namely, the wish to use equal velocity-pressure interpolations and to deal with convectiondominated flows. As it is now well known, both sources of instability can be overcome by using stabilized formulations. However, the main interest of this paper is not to explain how the stabilized formulation employed here allows to use equal interpolations or is able to avoid convection instabilities. Our main concern is to explain how to consider dynamic subscales (DS), how to integrate them in time and how to track them along the iterative process, accounting in particular for the

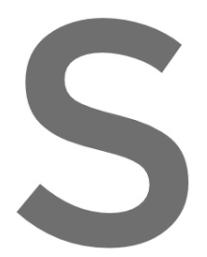
coupling of heat and

The paper is organi basic features. This se of the formulation to of the formulation. In p process, some possibilities to intr the cost of their storage. Two numerical examples are presented in Section 4, both of them are

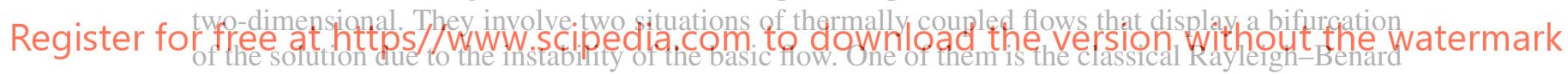
instability coupled with a Poiseuille flow, which leads to a transient flow even if the bifurcation is of stationary type. The second exampie is the classical fiow in a cavity with differentialily heated vertical walls. When the Prandtl number is small, the flow exhibits a Hopf bifurcation that leads to an oscillating flow pattern. The paper concludes in Section 5 with some final remarks and comments.

\section{THE NUMERICAL MODEL}

\subsection{Continuous problem}

Let $\Omega \subset \mathbb{R}^{d}$, with $d=2,3$, be the computational domain in which the flow takes place during the time interval $[0, T]$, and let $\Gamma$ be its boundary. The initial and boundary value problem to be considered consists in finding a velocity field $\mathbf{u}$, a pressure $p$ and a temperature $\vartheta$ such that

$$
\begin{aligned}
\partial_{t} \mathbf{u}+\mathbf{u} \cdot \nabla \mathbf{u}-v \Delta \mathbf{u}+\nabla p+\alpha \mathbf{g} \vartheta & =\mathbf{f}+\alpha \mathbf{g} \vartheta_{0} \quad \text { in } \Omega, t \in(0, T) \\
\nabla \cdot \mathbf{u} & =0 \quad \text { in } \Omega, t \in(0, T) \\
\partial_{t} \vartheta+\mathbf{u} \cdot \nabla \vartheta-\kappa \Delta \vartheta & =Q \quad \text { in } \Omega, t \in(0, T)
\end{aligned}
$$




$$
\begin{aligned}
& \mathbf{u}=\mathbf{0} \quad \text { on } \Gamma, t \in(0, T) \\
& \mathbf{u}=\mathbf{u}^{0} \quad \text { in } \Omega, t=0 \\
& \vartheta=0 \quad \text { on } \Gamma, t \in(0, T) \\
& \vartheta=\vartheta^{0} \quad \text { in } \Omega, t=0
\end{aligned}
$$

In these equations, $v$ is the kinematic viscosity, $\kappa$ the thermal diffusivity, $\alpha$ the thermal expansion coefficient, $\mathbf{f}$ the external body forces, $\vartheta_{0}$ the reference temperature, $\mathbf{g}$ the gravity acceleration vector, $Q$ the heat source and $\mathbf{u}^{0}$ and $\vartheta^{0}$ the initial conditions for velocity and temperature, respectively. For simplicity in the exposition, we have assumed homogeneous Dirichlet boundary conditions for both velocity and temperature.

To define the functional setting, let $H^{1}(\Omega)$ be the space of functions such that they and their first derivatives belong to $L^{2}(\Omega)$ (that is, they are square integrable), and let $H_{0}^{1}(\Omega)$ be the subspace of functions in $H^{1}(\Omega)$ vanishing on the boundary. Let also $\mathbf{V}_{\mathrm{st}}=H_{0}^{1}(\Omega)^{d}, Q_{\mathrm{st}}=L^{2}(\Omega) / \mathbb{R}$, $\Psi_{\text {st }}=H_{0}^{1}(\Omega)$ and define $\mathbf{V}=\mathbf{L}^{2}\left(0, T ; \mathbf{V}_{\text {st }}\right), Q=L^{1}\left(0, T ; Q_{\text {st }}\right)$ (for example) and $\Psi=L^{2}(0, T$; $\left.\Psi_{\text {st }}\right)$, where $L^{p}(0, T ; X)$ stands for the space of functions such that their $X$ norm in the spatial argument is an $L^{p}(0, T)$ function in time, that is, its $p$ th power is integrable if $1 \leqslant p<\infty$ or bounded if $p=\infty$.
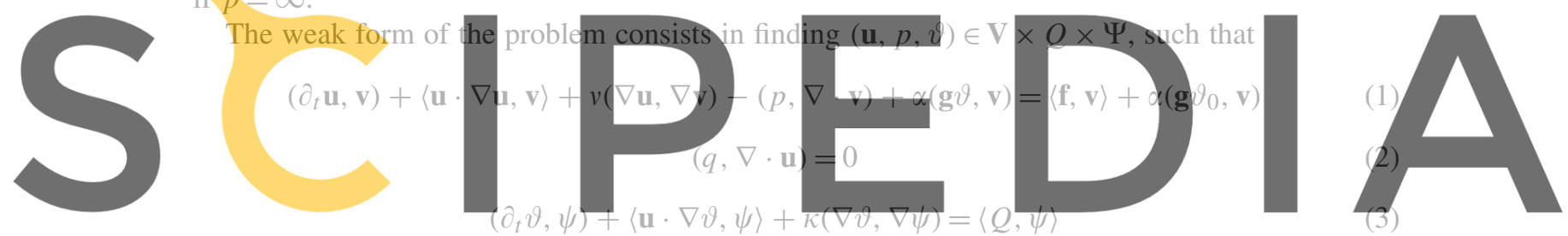

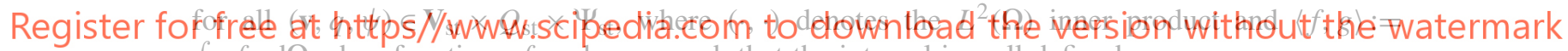
$\int_{\Omega} f g \mathrm{~d} \Omega$ when functions $f$ and $g$ are such that the integral is well defined.

The dimensionless numbers relevant to this problem are

$$
\begin{aligned}
& R e:=\frac{L U}{v} \quad \text { Reynolds number } \\
& P e:=\frac{L U}{\kappa} \quad \text { Péclet number } \\
& G r:=\frac{\alpha|\mathbf{g}| L^{3} \delta \vartheta}{v^{2}} \quad \text { Grashof number } \\
& \operatorname{Pr}:=\frac{v}{\kappa} \quad \text { Prandtl number } \\
& \operatorname{Ra}:=\frac{\alpha|\mathbf{g}| L^{3} \delta \vartheta}{v \kappa} \quad \text { Rayleigh number } \\
& F r:=\frac{U^{2}}{|\mathbf{g}| \delta \vartheta L} \quad \text { Froude number }
\end{aligned}
$$


where $L$ is the characteristic length of the problem, $U$ the characteristic velocity and $\delta \vartheta$ the characteristic temperature difference. When $U$ cannot be determined by the boundary conditions, for example, because zero velocities are prescribed, $U=v / L$ can be taken. These numbers are related by $R a=G r P r, F r=R e^{2} G r^{-1}$ and $R e=P e P^{-1}$.

\subsection{Scale splitting and space-discretized problem}

Let us consider a finite-element partition $\left\{\Omega_{e}\right\}, e=1, \ldots, n_{\mathrm{e}}$, of the computational domain $\Omega$, from which we can construct finite-element spaces for velocity, pressure and temperature in the usual manner. We will denote them by $\mathbf{V}_{h} \subset \mathbf{V}_{\text {st }}, Q_{h} \subset Q_{\text {st }}$ and $\Psi_{h} \subset \Psi_{\text {st }}$, respectively, and, to simplify the exposition, we will assume that they are all built from continuous piecewise polynomials of the same degree $k$.

The basic idea of the multiscale approach, we will follow [1], is to split the continuous unknowns as

$$
\begin{aligned}
& \mathbf{u}=\mathbf{u}_{h}+\tilde{\mathbf{u}} \\
& p=p_{h}+\tilde{p} \\
& \vartheta=\vartheta_{h}+\tilde{\vartheta}
\end{aligned}
$$

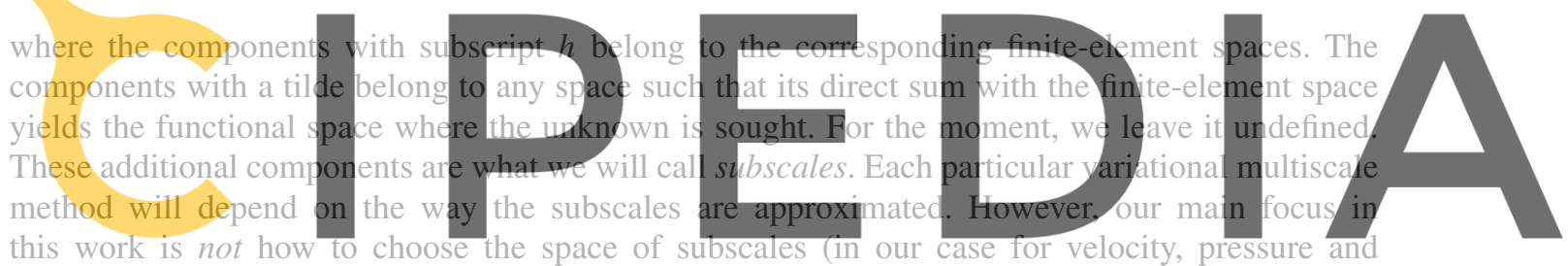

temperature), but to explain the consequences of considering these subscales time dependent, and

(4)-(6) in all the terms of (1)-(3). The only approximation we will make for the moment is to assume that the subscales vanish on the interelement boundaries, $\partial \Omega_{e}$. This happens, for example, if they are approximated using bubble functions [3], or if one assumes that their Fourier modes correspond to high wave numbers, as it is explained in [4].

Substituting (4)-(6) into (1)-(3), taking the test functions in the corresponding finite-element spaces and integrating some terms by parts, and using the fact that $\mathbf{u}=\mathbf{u}_{h}+\tilde{\mathbf{u}}$ is divergence free, it is found that

$$
\begin{gathered}
\left(\partial_{t} \mathbf{u}_{h}, \mathbf{v}_{h}\right)+\left\langle\mathbf{u}_{h} \cdot \nabla \mathbf{u}_{h}, \mathbf{v}_{h}\right\rangle+v\left(\nabla \mathbf{u}_{h}, \nabla \mathbf{v}_{h}\right)-\left(p_{h}, \nabla \cdot \mathbf{v}_{h}\right)+\alpha\left(\mathbf{g} \vartheta_{h}, \mathbf{v}_{h}\right) \\
-\left\langle\tilde{\mathbf{u}}, v \Delta_{h} \mathbf{v}_{h}+\mathbf{u}_{h} \nabla \cdot \mathbf{v}_{h}\right\rangle+\left(\partial_{t} \tilde{\mathbf{u}}, \mathbf{v}_{h}\right)+\left\langle\tilde{\mathbf{u}} \cdot \nabla \mathbf{u}_{h}, \mathbf{v}_{h}\right\rangle-\left\langle\tilde{\mathbf{u}}, \tilde{\mathbf{u}} \cdot \nabla \mathbf{v}_{h}\right\rangle \\
-\left(\tilde{p}, \nabla \cdot \mathbf{v}_{h}\right)+\alpha\left(\mathbf{g} \tilde{\vartheta}, \mathbf{v}_{h}\right)=\left\langle\mathbf{f}, \mathbf{v}_{h}\right\rangle+\alpha\left(\mathbf{g} \vartheta_{0}, \mathbf{v}_{h}\right) \\
\left(q q_{h}, \nabla \cdot \mathbf{u}_{h}\right)-\left(\tilde{\mathbf{u}}, \nabla q_{h}\right)=0 \\
\left(\partial_{t} \vartheta_{h}, \psi_{h}\right)+\left\langle\mathbf{u}_{h} \cdot \nabla \vartheta_{h}, \psi_{h}\right\rangle+\kappa\left(\nabla \vartheta_{h}, \nabla \psi_{h}\right)-\left\langle\tilde{\vartheta}, \kappa \Delta_{h} \vartheta_{h}+\mathbf{u}_{h} \cdot \nabla \psi_{h}\right\rangle \\
+\left(\partial_{t} \tilde{\vartheta}, \psi_{h}\right)+\left\langle\tilde{\mathbf{u}} \cdot \nabla \vartheta_{h}, \psi_{h}\right\rangle-\left\langle\tilde{\vartheta}, \tilde{\mathbf{u}} \cdot \nabla \psi_{h}\right\rangle=\left\langle Q, \psi_{h}\right\rangle
\end{gathered}
$$


which must hold for all test functions $\left(\mathbf{v}_{h}, q_{h}, \psi_{h}\right) \in \mathbf{V}_{h} \times Q_{h} \times \Psi_{h}$. The subindex $h$ in the Laplacian denotes that it is evaluated elementwise.

The first row in (7) corresponds to the terms arising from the classical Galerkin approximation of the momentum equation (except for the term due to external forces). Once the velocity subscale is approximated, the first term in the second row provides additional terms that appear in classical stabilized finite-element methods (see, for example, [5]). The rest of the terms are non-standard, in the sense that they are usually neglected. One of our purposes here is to discuss the implications of these terms. The last row in (7) comes from the contribution of pressure and temperature subscales and the contribution from the external forces. It is rather standard to take the pressure subscale into account, but to study the effect of the temperature subscale is one of the objectives of one of our numerical experiments.

In the left-hand side of (8) the first term is the classical Galerkin contribution, whereas the second provides (pressure) stability once the velocity subscale is approximated.

Similar comments to those made for (7) apply to (9). The first three terms of the first row correspond to the classical Galerkin approximation (except for the heat source), the last term of the first row provides stability in convection-dominated flows when the temperature subscale is approximated and, finally, the three terms in the left-hand side of the second row are non-standard, and come from the fact that subscales are never neglected in the previous equations (except for the fact that they are assumed to vanish on the interelement boundaries, as it has been already

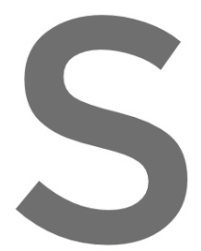
mentioned).

\section{Equations (7)-(9) c} element spaces of veloci by projecting onto the subscales instead of in of the subscale spaces (
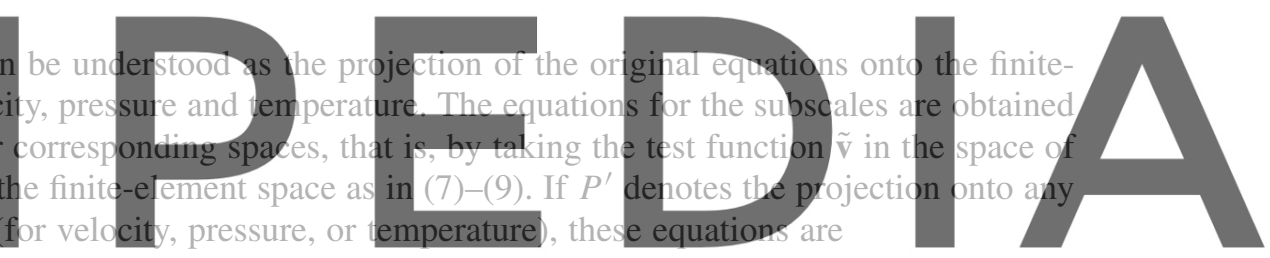

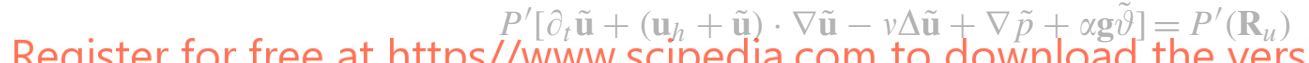

Register for free at https//wWw.scipedia.com to download the version without the watermark $P^{\prime}(\nabla \cdot \tilde{\mathbf{u}})=P^{\prime}\left(R_{p}\right)$

$$
P^{\prime}\left[\partial_{t} \tilde{\vartheta}+\left(\mathbf{u}_{h}+\tilde{\mathbf{u}}\right) \cdot \nabla \tilde{\vartheta}-\kappa \Delta \tilde{\vartheta}\right]=P^{\prime}\left(R_{\vartheta}\right)
$$

where

$$
\begin{aligned}
& \mathbf{R}_{u}=\mathbf{f}+\alpha \mathbf{g} \vartheta_{0}-\left[\partial_{t} \mathbf{u}_{h}+\left(\mathbf{u}_{h}+\tilde{\mathbf{u}}\right) \cdot \nabla \mathbf{u}_{h}-v \Delta_{h} \mathbf{u}_{h}+\nabla p_{h}+\alpha \mathbf{g} \vartheta_{h}\right] \\
& R_{p}=-\nabla \cdot \mathbf{u}_{h} \\
& R_{\vartheta}=Q-\left[\partial_{t} \vartheta_{h}+\left(\mathbf{u}_{h}+\tilde{\mathbf{u}}\right) \cdot \nabla \vartheta_{h}-\kappa \Delta_{h} \vartheta_{h}\right]
\end{aligned}
$$

are the residuals of the finite-element unknowns in the momentum, continuity and heat equation, respectively. Equations (10)-(12) need to be solved within each element and, as we have assumed, considering homogeneous velocity and temperature Dirichlet boundary conditions. Note that (7)-(9) could have been written in a similar way just replacing $P^{\prime}$ by $P_{h}$, the projection onto the appropriate finite-element space, and changing the role of subscales and finite-element unknowns.

It is not our purpose here to discuss how to approximate (10)-(11) which, in fact, is the essence of the different stabilized finite-element methods that can be found in the literature. We will adopt a simple approximation that can be found, for example, in [4] and references therein. Our main 
concern, as in the reference just mentioned, is to keep the time dependence of the subscales, as well their nonlinear effects. When their time derivative is neglected, we will call them quasistatic, whereas otherwise we will call them dynamic.

Using the same arguments as in [4], now extended to thermally coupled flows, we propose to compute the subscales within each element of the finite-element partition as solution to

$$
\begin{gathered}
\partial_{t} \tilde{\mathbf{u}}+\frac{1}{\tau_{1}} \tilde{\mathbf{u}}=P^{\prime}\left(\mathbf{R}_{u}\right) \\
\frac{1}{\tau_{2}} \tilde{p}=P^{\prime}\left(R_{p}+\tau_{1} \partial_{t} R_{p}\right) \\
\partial_{t} \tilde{\vartheta}+\frac{1}{\tau_{3}} \tilde{\vartheta}=P^{\prime}\left(R_{\vartheta}\right)
\end{gathered}
$$

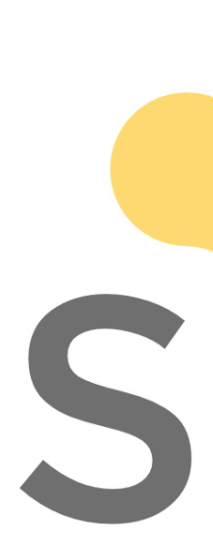

where the stabilization parameters $\tau_{1}, \tau_{2}$, and $\tau_{3}$ are computed as

$$
\tau_{1}=\left(c_{1} \frac{v}{h^{2}}+c_{2} \frac{\left|\mathbf{u}_{h}+\tilde{\mathbf{u}}\right|}{h}\right)^{-1}
$$
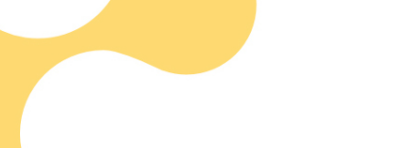

Where $h$ is the element
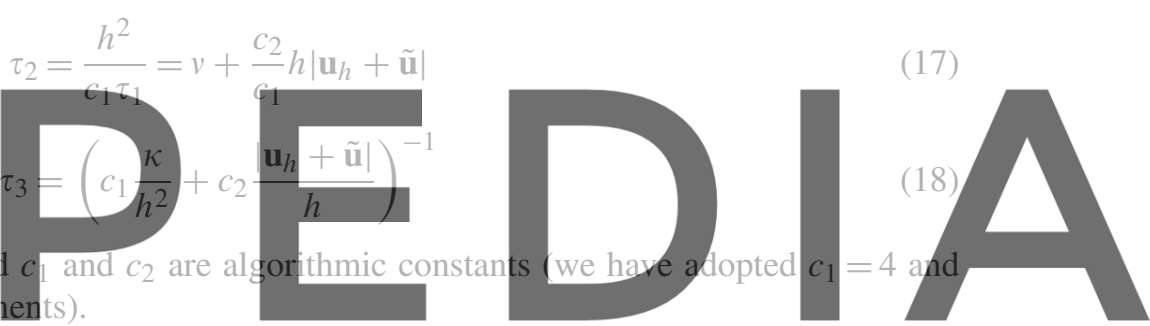

The approximation adopted for the subscales could certainly be improved, for example, by trying

another. However, our interest here is only to analyse the effect of considering the subscales time

dependent and taking into account their contribution in the nonlinear terms. In particular, it is important to remark that (13) is nonlinear, both because the velocity subscale contributes to the advection velocity and because the stabilization parameter $\tau_{1}$ depends also on the velocity subscale, as Equation (15) and the stabilization parameter $\tau_{3}$. Likewise, (13) depends on the temperature subscale, and therefore the velocity-temperature coupling is naturally accounted for.

Even though it is not our purpose to use an 'accurate' approximation to the subscales, in some cases we have found convenient to include the time derivative of $R_{p}$ in the approximation (14) of the pressure subscale. This term was neglected in [4], but in some situations it is crucial to improve pressure stability. This time derivative arises naturally if one takes the divergence of the exact momentum equation to obtain a pressure Poisson equation for the pressure subscale and then approximates this pressure equation within each element.

It is observed that in (13)-(15) we have kept the projections $P^{\prime}$ in the right-hand side terms. Basically, two different options can be considered. Classical stabilized finite-element methods are recovered by taking $P^{\prime}=I$ (the identity), whereas if $P^{\prime}=P_{h}^{\perp}=I-P_{h}, P_{h}$ being the $L^{2}$ projection onto the appropriate finite-element space, the subscales turn out to be orthogonal to this finite-element space. The resulting formulation is termed as orthogonal subscales stabilization in [4]. 
The space-discrete formulation is now complete. However, contrary to what happens with quasistatic subscales (QSS) and neglecting their nonlinear effects, now it is not possible to obtain a closed-form expression for these subscales and insert them into (7)-(9) in order to obtain a problem for the finite-element components of velocity, pressure, and temperature. Prior to discretizing in time, we cannot go any further than saying that the problem consists in solving (7)-(9) together with (13)-(15).

\subsection{Discretization in time}

Any finite-difference scheme can now be applied to discretize in time both in Equations (7)-(9) and (13)-(15). Obviously, space-time finite-element discretizations are also possible. In order to make the exposition concise, we will restrict our attention to the trapezoidal rule.

Let $\delta t$ be the time step size of a uniform partition of the time interval $[0, T], 0=t^{0}<t^{1}<\cdots<t^{N}=$ $T$. Functions approximated at time $t^{n}$ will be identified with the superscript $n$. For a generic function $f$, we will use the notation $\delta f^{n}:=f^{n+1}-f^{n}, \delta_{t} f^{n}=\delta f^{n} / \delta t, f^{n+\beta}=\beta f^{n+1}+(1-\beta) f^{n}$, $0 \leqslant \beta \leqslant 1$.

The time discretization of (7)-(9) is standard and does not need any further explanation. Given $\mathbf{u}_{h}^{n}, \vartheta_{h}^{n}, \tilde{\mathbf{u}}^{n}$, and $\tilde{\vartheta}^{n}$, it consists of solving the problem
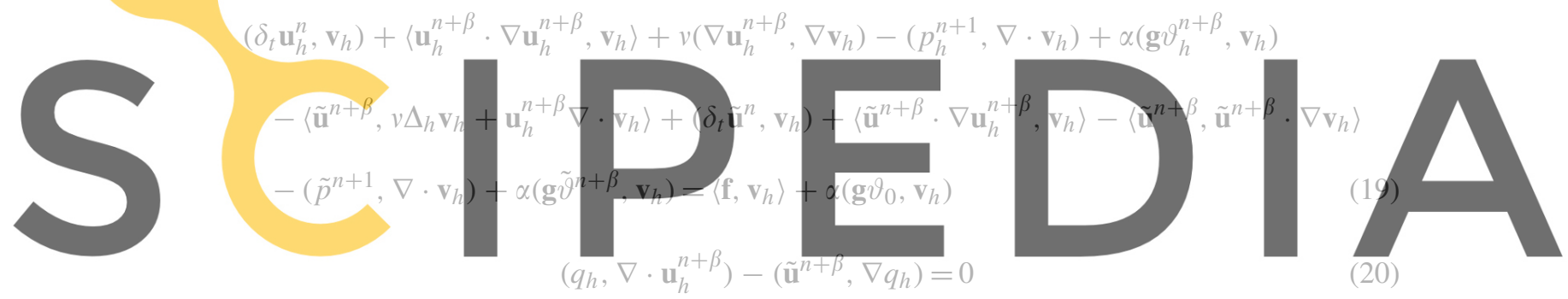

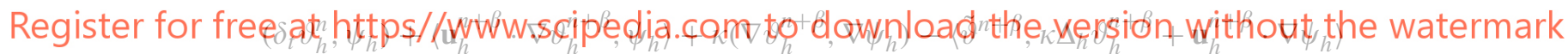

$$
+\left(\delta_{t} \tilde{\vartheta}^{n}, \psi_{h}\right)+\left\langle\tilde{\mathbf{u}}^{n+\beta} \cdot \nabla \vartheta_{h}^{n+\beta}, \psi_{h}\right\rangle-\left\langle\tilde{\vartheta}^{n+\beta}, \tilde{\mathbf{u}}^{n+\beta} \cdot \nabla \psi_{h}\right\rangle=\left\langle Q, \psi_{h}\right\rangle
$$

which must hold for all test functions $\left(\mathbf{v}_{h}, q_{h}, \psi_{h}\right) \in \mathbf{V}_{h} \times Q_{h} \times \Psi_{h}$. Note that the pressure is considered approximated at time $n+1$. This avoids the need to deal with the pressure at a previous time step and does not modify the velocity approximation. As it is well known, the scheme is expected to be of second-order if $\beta=\frac{1}{2}$ and of first-order otherwise.

Equations (13)-(15) need also to be integrated in time. The simplest option is to use the same time discretization as for the finite-element equations, which yields

$$
\begin{gathered}
\delta_{t} \tilde{\mathbf{u}}^{n}+\frac{1}{\tau_{1}^{n+\beta}} \tilde{\mathbf{u}}^{n+\beta}=P^{\prime}\left(\mathbf{R}_{u}^{n+\beta}\right) \\
\frac{1}{\tau_{2}^{n+1}} \tilde{p}^{n+1}=P^{\prime}\left(R_{p}^{n+1}+\tau_{1}^{n+1} \delta_{t} R_{p}^{n}\right) \\
\delta_{t} \tilde{\vartheta}^{n}+\frac{1}{\tau_{3}^{n+\beta}} \tilde{\vartheta}^{n+\beta}=P^{\prime}\left(R_{\vartheta}^{n+\beta}\right)
\end{gathered}
$$


However, we will consider two additional options. The first is that the time integration for the subscales could be less accurate than for the finite-element equations (7)-(9) and still keep the same order of accuracy in time of the finite-element solution. The formal idea to justify this is the following. From the expression of the stabilization parameters $\tau_{1}$ and $\tau_{3}$ in (16) and (18), respectively, it follows that they behave as the critical time steps of an explicit integration in time of the momentum and the heat equation [6]. Therefore, we may assume that they are of order $\mathcal{O}(\delta t)$. From (22) it follows that $\mathcal{O}(1) \delta \tilde{\mathbf{u}}^{n+1}+\tilde{\mathbf{u}}^{n+1}=\mathcal{O}(\delta t) P^{\prime}\left(\mathbf{R}_{u}^{n+\beta}\right)$, and thus we may conclude that $\tilde{\mathbf{u}}^{n+1}=\mathcal{O}(\delta t) P^{\prime}\left(\mathbf{R}_{u}^{n+1}\right)$. If the residual of the finite-element component is bounded, $\left|\tilde{\mathbf{u}}^{n+1}-\tilde{\mathbf{u}}^{n}\right|=\mathcal{O}\left(\delta t^{2}\right)$, and therefore evaluating the subscale at $n+1$, for example, in (19) instead of at $n+\beta$ introduces an error of order $\mathcal{O}\left(\delta t^{2}\right)$, which is the optimal error that can be reached with the trapezoidal rule (for $\beta=\frac{1}{2}$ ). The same comments apply to (24) for the temperature subscale.

Considering the subscale equations integrated to first-order and the finite-element equations to second (or higher) is not particularly relevant in the case of the trapezoidal rule. However, if, for example, the second-order backward-differencing scheme is used, a first-order integration of the equation for the subscales avoids the need to store them in two previous time steps. This storage is the most important cost of integrating the subscales in time. Another aspect to take into account is that the subscale approximation is not smooth, since the residual of the finite-element components will be discontinuous across interelement boundaries. Thus, it seems reasonable to use a scheme

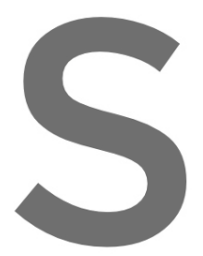
as dissipative as possible to integrate the sybscales in time. Further comments about this point are
made in Section 4 .
A first-order time integration for the subscales is straightforward. Equations (22) and (24) have
to be replaced by their counterparts for $\beta-1$.
A third and final possibility that can be considered to integrate (13)-(15) in time is a combination
of exact integration and approx mation of the stabilization narameters and residuals at $n+\beta$. If his of exact integration and approximation of the stabilization parameters and residuals at
approximation is done, the equations for the velocity and temperature subscales are

$$
\begin{aligned}
& \partial_{t} \widetilde{\mathbf{u}}+\frac{\tau_{1}^{n+\beta}}{\tau_{1}}=P^{\prime}\left(\mathbb{R}_{u}^{n}\right) \\
& \partial_{t} \tilde{\vartheta}+\frac{1}{\tau_{3}^{n+\beta}} \tilde{\vartheta}=P^{\prime}\left(R_{\vartheta}^{n+\beta}\right)
\end{aligned}
$$

which can be integrated exactly, yielding

$$
\begin{aligned}
& \tilde{\mathbf{u}}^{n+1}=\left(\tilde{\mathbf{u}}^{n}-\tau_{1}^{n+\beta} P^{\prime}\left(\mathbf{R}_{u}^{n+\beta}\right)\right) \exp \left(-\frac{\delta t}{\tau_{1}^{n+\beta}}\right)+\tau_{1}^{n+\beta} P^{\prime}\left(\mathbf{R}_{u}^{n+\beta}\right) \\
& \tilde{\vartheta}^{n+1}=\left(\tilde{\vartheta}^{n}-\tau_{3}^{n+\beta} P^{\prime}\left(R_{\vartheta}^{n+\beta}\right)\right) \exp \left(-\frac{\delta t}{\tau_{3}^{n+\beta}}\right)+\tau_{3}^{n+\beta} P^{\prime}\left(R_{\vartheta}^{n+\beta}\right)
\end{aligned}
$$

\subsection{Main features of the formulation}

The method described so far is an extension of the formulation proposed in [2] to the case of thermally coupled flows using the Boussinesq approximation. Even though the main contribution of this paper is this extension and the discussion of Section 3 about some implementation aspects, 
let us recall some of the fundamental features of the formulation already discussed in [2]. All what we say for the velocity subscale also applies to the temperature subscale, except if it only makes sense in the first case.

2.4.1. Time integration properties. The first and most important point to be considered is the effect of considering the subscales dynamic, and therefore to deal with their time variation. Some of these properties are:

- The effect of the time integration is now clear. Suppose, for example, that we are using (22)-(24) to integrate the subscales. Certainly, the effective stabilization parameters have to be modified (as it is done, for example, in $[7,8]$ ), but when the steady state is reached the subscale $\tilde{\mathbf{u}}$ that is obtained satisfies

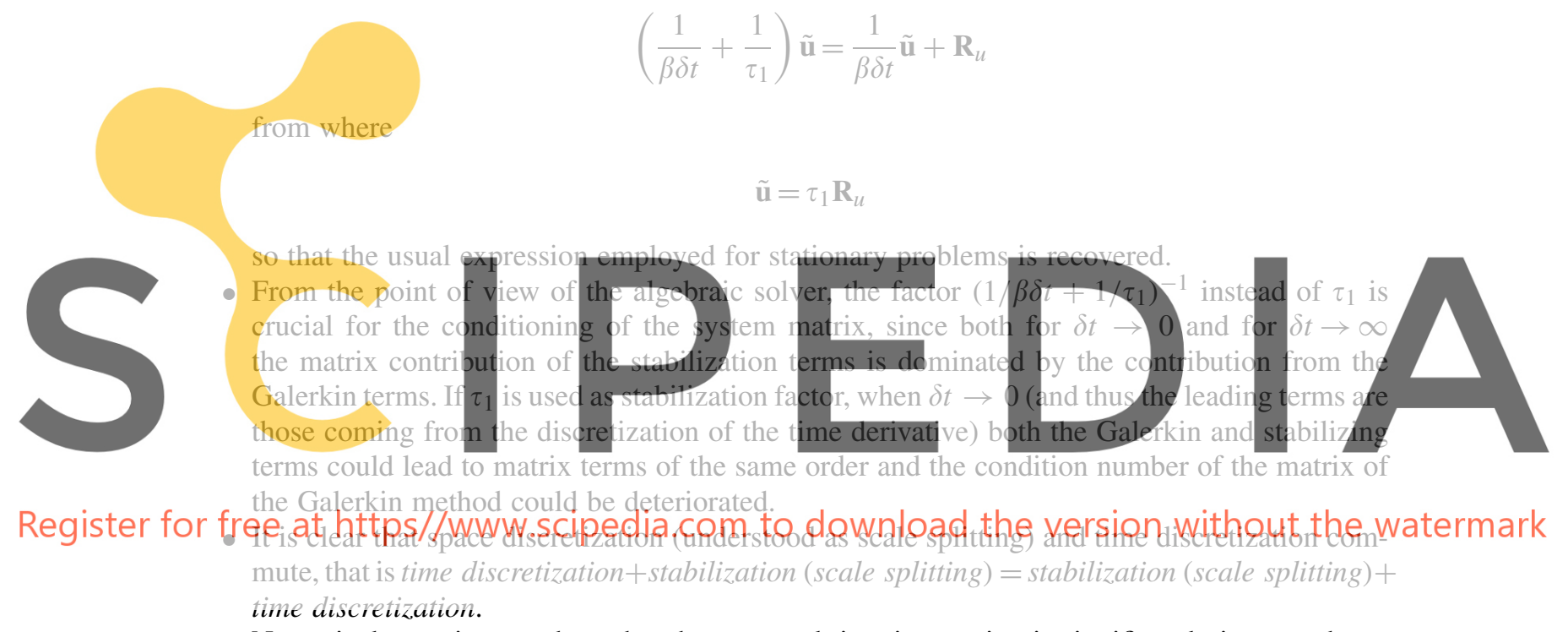

- Numerical experiments show that the temporal time integration is significantly improved:

- Oscillations originated by initial transients are eliminated.

○ The numerical dissipation is minimized.

For the numerical results that demonstrate this fact we refer to [2]. This is also observed in the numerical experiments of Section 4.

- The numerical analysis shows optimal stability without any restriction between $\tau_{1}$ and $\delta t$. Contrary to classical stabilized methods, anisotropic space-time discretizations are allowed [9]. See [2] for a stability analysis of the linearized Navier-Stokes equations and [10] for a complete stability and convergence analysis for the Stokes problem.

2.4.2. Global momentum conservation. This is provided by the term $\left\langle\tilde{\mathbf{u}}^{n+\beta} \cdot \nabla \mathbf{u}_{h}^{n+\beta}, \mathbf{v}_{h}\right\rangle$. It can be shown that global momentum conservation holds if [11]

$$
-\int_{\Omega} u_{h, 1}^{n+\beta} \nabla \cdot \mathbf{u}_{h}^{n+\beta} \mathrm{d} \Omega+\int_{\Omega} \tilde{\mathbf{u}}^{n+\beta} \cdot \nabla u_{h, 1}^{n+\beta} \mathrm{d} \Omega=0
$$


This is implied by the continuity equation

$$
\left(q_{h}, \nabla \cdot \mathbf{u}_{h}^{n+\beta}\right)-\left(\tilde{\mathbf{u}}^{n+\beta}, \nabla q_{h}\right)=0
$$

provided $V_{h, 1} \subseteq Q_{h}$. This holds, in particular, for the 'natural' choice $V_{h, 1}=Q_{h}$. For the standard Galerkin method, this condition is impossible to be satisfied, since equal interpolation does not satisfy the inf-sup condition. As a conclusion, the term $\left\langle\tilde{\mathbf{u}}^{n+\beta} \cdot \nabla \mathbf{u}_{h}^{n+\beta}, \mathbf{v}_{h}\right\rangle$ provides global momentum conservation.

2.4.3. About the possibility to model turbulence. The term $\left\langle\tilde{\mathbf{u}}^{n+\beta}, \tilde{\mathbf{u}}^{n+\beta} \cdot \nabla \mathbf{v}_{h}\right\rangle=\left\langle\nabla \mathbf{v}_{h}, \tilde{\mathbf{u}}^{n+\beta} \otimes\right.$ $\left.\tilde{\mathbf{u}}^{n+\beta}\right\rangle$ can be understood as the contribution from the residual stress tensor or the subgrid scale tensor of a large eddy simulation approach. Therefore, we may expect that, in some sense, modelling $\tilde{\mathrm{u}}^{n+\beta}$ implies to model the subgrid scale tensor. The question is how good this model will be. The numerical models proposed here yield two possibilities, but many others can be devised. Some of the questions we should pose to any model could be:

- Is the Kolmogorov energy cascade properly reproduced? This implies that the kinetic energy in the Fourier space
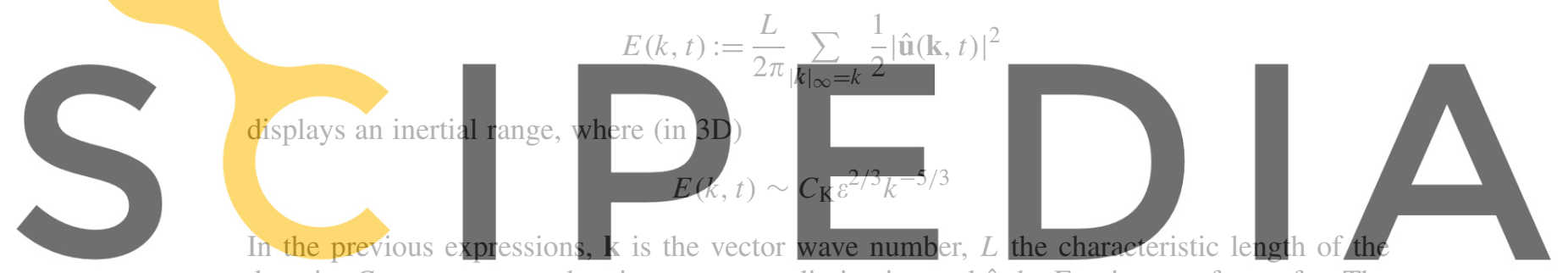

domain, $C_{\mathrm{K}}$ a constant, $\varepsilon$ the viscous energy dissipation and $\hat{\mathbf{u}}$ the Fourier transform of u. The

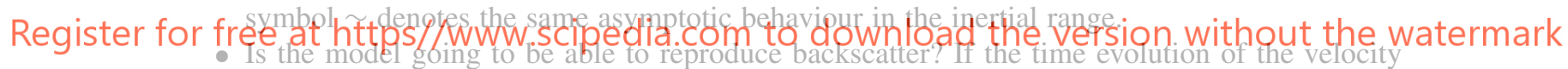

subscale and its nonlinear effects are not taken into account this is not possible, since the additional terms introduced by classical stabilized methods can be shown to produce always energy dissipation.

- Is the dimension of the global attractor properly reproduced? The heuristic estimate is

$$
\mathscr{N} \sim\left(\frac{L}{\lambda_{\mathrm{K}}}\right)^{3} \sim R e^{9 / 4}
$$

where $\lambda_{\mathrm{K}}$ is the Kolmogorov length scale and Re the Reynolds number. The closest estimate analytically proved is (roughly) $\left(L / \lambda_{\mathrm{K}}\right)^{4.8}[12]$.

- Are turbulent boundary layers properly approximated? According to Prandtl theory, this implies a log behaviour right after the laminar sublayer. Likewise, are shear layers properly reproduced?

- Is the energy decay in time correctly captured? And, in particular, in a homogeneous isotropic turbulence, is relaminarization properly predicted?

These and other properties should be expected from a numerical model that intends to model turbulent flows. The answer to all these questions deserves further research, both of numerical and of theoretical nature. Some of the results presented in [2] seem to indicate that in fact it is possible to model turbulent flows with the numerical approach proposed here, or even with simplified versions 
that can be found in some recent works (see, for example, [13-15] or the review [16] and references therein). Another important point is that the formulation we propose would account for thermal turbulence in a natural way. The traditional approach is to relate thermal turbulence to the mechanical one through the introduction of a turbulent Prandtl number whose physical meaning and adequate value are not well understood. This would be unnecessary with the approach presented here.

\section{SOME COMPUTATIONAL ASPECTS}

The purpose of this section is to discuss some of the implementation aspects of the numerical formulation presented before. In particular, we will concentrate on three points:

- A common format for the different time integration options discussed for the subscales.

- How to deal with the additional nonlinearity introduced by taking into account the nonlinear effects of the subscales.

- Which simplifications could be done to reduce the storage of the subscales.

\subsection{Time integration schemes for the subscales}

In Section 2, we have presented three different alternatives to integrate the subscales in time, namely, the same time integration as for the finite-element component given by (22)-(24), a first-order time integration obtained by taking $\beta=1$ and the approximation given by (25)-(26). All these schemes can be written as

$$
\tilde{w}^{n+1}(\mathbf{x})=\xi^{n+1}(\mathbf{x}) \tilde{w}^{n}(\mathbf{x})+\eta^{n+1}(\mathbf{x}) P^{\prime}\left(R^{n+1}\right)(\mathbf{x})
$$

where $w$ is the unknown under consideration (velocity or temperature), $R$ is the corresponding residual of the finite-element equation and the coefficients $\xi$ and $\eta$ depend on the time integration scheme used. Their expression is given in Table I, where $\tau$ denotes the appropriate stabilization parameter (for velocity or for temperature). Coefficients $\xi$ and $\eta$ depend on the spatial point and the time step level through this parameter, which in turn depends on the subscale $\tilde{w}$ and on $w_{h}$, the finite-element component of $w$. Thus, (27) is a nonlinear algebraic equation whose linearization is discussed in Section 3.2.

\subsection{Linearization}

Let us consider (27) with $R^{n+1}=f^{n+1}-\mathscr{L}^{n+1} w_{h}^{n+1}$, where $f$ is the source term of the equation under consideration (velocity or temperature) including contributions from the time integration,

Table I. Coefficients of the time integration schemes for the subscales.

\begin{tabular}{lcc}
\hline Method & $\xi$ & $\eta$ \\
\hline First order & $\frac{\tau}{\tau+\delta t}$ & $\frac{\tau \delta t}{\tau+\delta t}$ \\
Trapezoidal rule & $\frac{\tau-(1-\beta) \delta t}{\tau+\beta \delta t}$ & $\frac{\tau \delta t}{\tau+\beta \delta t}$ \\
Exact & $\exp \left(-\frac{\delta t}{\tau}\right)$ & $\tau\left[1-\exp \left(-\frac{\delta t}{\tau}\right)\right]$ \\
\hline
\end{tabular}


$\mathscr{L}^{n+1}$ is the differential operator (in general nonlinear) of this equation and $w_{h}$ is the finite-element component of unknown $w$. As it has been mentioned, the coefficients $\xi$ and $\eta$ depend on both $\tilde{w}$ and $w_{h}$ through the stabilization parameter $\tau$. We may explicitly write these dependencies as

$$
\tilde{w}^{n+1}=\xi\left(\tilde{w}^{n+1}, w_{h}^{n+1}\right) \tilde{w}^{n}+\eta\left(\tilde{w}^{n+1}, w_{h}^{n+1}\right) P^{\prime}\left[f^{n+1}-\mathscr{L}\left(\tilde{w}^{n+1}, w_{h}^{n+1}\right) w_{h}^{n+1}\right]
$$

Given a guess for the subscale, the linearization of the equation for the finite-element component is standard and will not be discussed here. Any option is possible. We assume that the $i$ th iterate of $w_{h}$ in the time step $n+1$, denoted by $w_{h}^{n+1, i}$, has been computed using a guess $\tilde{w}^{n+1, i-1}$ for the subscale, and now the goal is to obtain a new guess for the subscale, $\tilde{w}^{n+1, i}$. To that end, we use a simple fixed point scheme. Within each iteration of the loop to update the finite-element unknown, we solve until convergence

$$
\begin{aligned}
\tilde{w}^{n+1, i, j}= & \xi\left(\tilde{w}^{n+1, i, j-1}, w_{h}^{n+1, i}\right) \tilde{w}^{n}+\eta\left(\tilde{w}^{n+1, i, j-1}, w_{h}^{n+1, i}\right) \\
& \times P^{\prime}\left[f^{n+1}-\mathscr{L}\left(\tilde{w}^{n+1, i, j-1}, w_{h}^{n+1, i}\right) w_{h}^{n+1, i}\right]
\end{aligned}
$$

In this equation, $\tilde{w}^{n+1, i, j}$ denotes the subscale computed at the $j$ th iteration within the $i$-iteration to compute the finite-element unknown of time step $n+1$. As initial guess we take $\tilde{w}^{n+1, i, 0}=\tilde{w}^{n+1, i-1}$.

Relaxation can be applied to both the inner loop (with iteration counter $j$ ) and to the outer loop (with iteration counter $i$ ), so that if $\rho_{1}$ and $\rho_{2}$ are the relaxation parameters we may reset

$$
\begin{gathered}
\tilde{w}^{n+1, i, j} \leftarrow \rho_{1} \tilde{w}^{n+1, i, j}+\left(1-\rho_{1}\right) \tilde{w}^{n+1, i, j-1} \\
\tilde{w}^{n+1, i} \leftarrow \rho_{2} \tilde{w}^{n+1, i}+\left(1-\rho_{2}\right) \tilde{w}^{n+1, i-1}
\end{gathered}
$$

\subsection{Two simplifications}

The straightforward way to implement the formulation proposed is to evaluate and store the subscales at the integration points of the numerical integration rule employed to approximate the integrals. For each scalar subscale (velocity components or temperature) the values to be stored are those of the previous time step (in the case of the simplest one-step time integration schemes) and of the previous iteration. This implies an important amount of storage, similar to that required for internal variables in solid mechanics. In this subsection, we consider two alternatives to reduce this storage cost.

3.3.1. Underintegration of the subscales. It is well known that the numerical integration rule that needs to be used to integrate the Galerkin contribution in (19)-(21) is more accurate than the one that would be needed to preserve the spatial accuracy of the finite-element approximation. For example, when using finite-element interpolations of degree $k$, a numerical integration able to integrate exactly polynomials of degree $k$ would be enough to yield a spatial error of degree $k+1$ in $L^{2}(\Omega)$, but this may cause either rank deficiency of some of the matrix contributions or a poor response in time of the time integration scheme employed [17]. This is why these, in principle, sufficient integration rules are termed underintegration. However, there is no reason 'a priori' to avoid them to integrate the terms that involve the subscales, particularly those that require their storage. 
3.3.2. Approximation based on nodal stabilization parameters. To simplify the exposition, let us assume that $P^{\prime} f=0$. The following approximation is based on taking a nodal value for the stabilization parameters, and therefore, for the coefficients $\xi$ and $\eta$ appearing in (27).

Let $N_{a}(\mathbf{x})$ be the shape function associated with node $a$ of the finite-element mesh and $W_{a}^{n}$ the nodal value at this node of the finite-element unknown $w_{h}$ at time step $n$. Here and below index $a$ runs everywhere from 1 to the number of mesh points, $n_{\mathrm{p}}$.

Let us assume that at time level $n$ the subscale $\tilde{w}^{n}$ can be expressed as

$$
\tilde{w}^{n}(\mathbf{x})=\sum_{a} \varphi_{a}^{n}(\mathbf{x}) \bar{W}_{a}^{n}+\sum_{a} \varphi_{a}^{n-1}(\mathbf{x}) \hat{W}_{a}^{n}
$$

for certain coefficients $\bar{W}_{a}^{n}$ and $\hat{W}_{a}^{n}$, and where

$$
\varphi_{a}^{n}(\mathbf{x}):=P^{\prime} \mathscr{L}^{n} N_{a}(\mathbf{x})
$$

Our aim is to show that (31) holds in an approximate sense also at time step $n+1$ and therefore, proceeding inductively, it will hold for all time steps. We will, in particular, make use of the approximation

$$
\varphi_{a}^{n-1}(\mathbf{x})=2 \varphi_{a}^{n}(\mathbf{x})-\varphi_{a}^{n+1}(\mathbf{x})+\mathcal{O}\left(\delta t^{2}\right)
$$

If (31) holds, it is clear that $\left\{\varphi_{a}^{n}(\mathbf{x}), \varphi_{a}^{n-1}(\mathbf{x})\right\}$ span the space of subscales. Obviously, these functions need not to be linearly independent.

Once the nonlinear equation (27) has been solved by the iterative procedure described earlier, we will have that

$$
\begin{aligned}
\tilde{w}^{n+1}(\mathbf{x})= & \xi^{n+1}(\mathbf{x}) \sum_{a}\left(\varphi_{a}^{n}(\mathbf{x}) \bar{W}_{a}^{n}+\varphi_{a}^{n-1}(\mathbf{x}) \hat{W}_{a}^{n}\right)-\eta^{n+1}(\mathbf{x}) \sum_{a} \varphi_{a}^{n+1}(\mathbf{x}) W_{a}^{n+1} \\
= & \sum_{a} \xi^{n+1}(\mathbf{x})\left(\bar{W}_{a}^{n}+2 \hat{W}_{a}^{n}\right) \varphi_{a}^{n}(\mathbf{x}) \\
& -\sum_{a}\left(\xi^{n+1}(\mathbf{x}) \hat{W}_{a}^{n}+\eta^{n+1}(\mathbf{x}) W_{a}^{n+1}\right) \varphi_{a}^{n+1}(\mathbf{x})+\mathcal{O}\left(\delta t^{2}\right)
\end{aligned}
$$

where we have made use of (33). The essential approximation, however, is the following. Since the support of $N_{a}(\mathbf{x})$ is local, each term within the summation is different from zero only in this local support. The coefficients $\xi^{n+1}(\mathbf{x})$ and $\eta^{n+1}(\mathbf{x})$ can be taken as constants in this support and equal nodal to the values $\xi_{a}^{n+1}$ and $\eta_{a}^{n+1}$, respectively. These nodal values can be computed from nodal averaging of the stabilization parameters on which they depend, or by a least-squares fitting to preserve the optimal approximation error in space. In the nonlinear scheme (28) this needs to be done at each iteration. Another possibility is to evaluate directly the stabilization parameters at the nodes [18].

Using (33) and the approximation just described, we have that

$$
\tilde{w}^{n+1}(\mathbf{x}) \approx \sum_{a} \xi_{a}^{n+1}\left(\bar{W}_{a}^{n}+2 \hat{W}_{a}^{n}\right) \varphi_{a}^{n}(\mathbf{x})-\sum_{a}\left(\xi_{a}^{n+1} \hat{W}_{a}^{n}+\eta_{a}^{n+1} W_{a}^{n+1}\right) \varphi_{a}^{n+1}(\mathbf{x})
$$

From this expression we see that (31) also holds (approximately) at time step $n+1$, with

$$
\bar{W}_{a}^{n+1}=-\xi_{a}^{n+1} \hat{W}_{a}^{n}-\eta_{a}^{n+1} W_{a}^{n+1}
$$




$$
\hat{W}_{a}^{n+1}=\xi_{a}^{n+1}\left(\bar{W}_{a}^{n}+2 \hat{W}_{a}^{n}\right)
$$

The importance of this result is clear. According to (31), it is not necessary to store the subscales at the integration points, but it is enough to store the two arrays $\left\{\bar{W}_{a}^{n}\right\}$ and $\left\{\hat{W}_{a}^{n}\right\}, a=1, \ldots, n_{\mathrm{p}}$. The savings, particularly in 3D cases, are notorious. For example, in 3D unstructured meshes of $P_{1}$ elements the ratio of elements to nodal points is about 6 , and full integration requires 4 points per element. Therefore, storing the subscales at the integration points would require 12 times the memory needed to store arrays $\left\{\bar{W}_{a}^{n}\right\}$ and $\left\{\hat{W}_{a}^{n}\right\}$.

Expression (31) together with Equations (34)-(35) to update the coefficients of (31) are enough if the operator $\mathscr{L}$ does not depend on the subscales. However, this is not the case, neither for the Navier-Stokes nor for the heat equations that we are considering. In the latter case, for example, and using the trapezoidal rule with $\beta=1$ to integrate in time

$$
\varphi_{a}^{n}(\mathbf{x})=P^{\prime} \mathscr{L}^{n} N_{a}(\mathbf{x})=P^{\prime}\left(\frac{1}{\delta t} N_{a}+\left(\mathbf{u}_{h}^{n}+\tilde{\mathbf{u}}^{n}\right) \cdot \nabla N_{a}-\kappa \Delta N_{a}\right)
$$

so that $\tilde{\mathbf{u}}^{n}$ is needed at the integration points.

Equation (31) has to be considered in fact a representation formula rather than an explicit expression for the subscales. At each integration point, it has to be understood as a nonlinear equation whose approximation can be naturally coupled to that of the subscale at time step $n+1$. If $\varphi_{a}^{n, i, j-1}(\mathbf{x})$ is the function in (32) evaluated with a guess $\tilde{w}^{n, i, j-1}$ of the subscale at the $i$ th iteration for the finite-element unknown of the $n+1$-time step, we may readapt the iterative procedure (28) to

$$
\begin{aligned}
\tilde{w}^{n, i, j}= & \sum_{a} \varphi_{a}^{n, i, j-1} \bar{W}_{a}^{n}+\sum_{a}\left(2 \varphi_{a}^{n, i, j-1}-\varphi_{a}^{n+1, i, j-1}\right) \hat{W}_{a}^{n} \\
\tilde{w}^{n+1, i, j}= & \xi\left(\tilde{w}^{n+1, i, j-1}, w_{h}^{n+1, i}\right) \tilde{w}^{n, i, j}+\eta\left(\tilde{w}^{n+1, i, j-1}, w_{h}^{n+1, i}\right) \\
& \times P^{\prime}\left[f^{n+1}-\mathscr{L}\left(\tilde{w}^{n+1, i, j-1}, w_{h}^{n+1, i}\right) w_{h}^{n+1, i}\right]
\end{aligned}
$$

where, once more, we have made use of approximation (33).

In the previous development we have considered that $P^{\prime} f=0$. If this is not the case, one can consider its expansion in a basis of the form $f(\mathbf{x})=\sum_{i} f_{i} \psi_{i}(\mathbf{x})$, and incorporate $P^{\prime} \psi_{i}(\mathbf{x})$ in the set of functions that span the space of subscales. Index $i$ runs up to the dimension of the space where the force term is defined, perhaps in an approximate manner.

\section{NUMERICAL EXAMPLES}

In this section, we present the results of two numerical tests involving two-dimensional thermally coupled flows. In both cases we have used $P^{\prime}=I$ in (10)-(12), which corresponds to the most classical stabilized finite-element methods.

In both numerical examples, our purpose is to compare the numerical performance of QSS and DS. To this end, we will proceed as follows. Two meshes will be considered in both examples, one that we will call 'coarse' and another finer one. On both meshes we will present the results obtained using QSS and, only in the coarse mesh, the results are obtained considering DS. The goal 
is to show that DS yield better results than QSS on the coarse meshes by comparing both to the QSS results on the fine mesh, that we will call reference results. We anticipate that the conclusions of the following numerical experiments are

- The accuracy is higher using DS. This is reflected, in particular, by less damping of frequencies and amplitudes in the oscillating response of the flows considered.

- Stability is improved by using DS, particularly when subscales are integrated in time with a first-order scheme. Some oscillations encountered with QSS are removed.

In all the runs the nonlinearity of the subscale has to be dealt with using the iterative scheme described in Section 3.2. When the flow is fully developed, it converges very well, yielding fully converged subscales (with relative residuals of the order of $10^{-6}$ ) with four or five iterations. The velocity-temperature coupling has been achieved using a block-iterative strategy, using also a nested iterative loop to solve the nonlinear Navier-Stokes equations within each coupling iteration. Concerning the time integration schemes, the equations for the finite-element unknowns have been integrated using the second-order Crank-Nicolson scheme $\left(\beta=\frac{1}{2}\right.$ in (19)-(21)), whereas the equations for the subscales have been integrated either using this same scheme or the first-order version described in Section 3.1.

\subsection{Thermoconvective instability of plane Poiseuille flow}

The problem consists of a two-dimensional laminar flow in a horizontal channel occupying the domain $[0,10] \times[0,1]$ and suddenly heated from below. A parabolic inlet velocity profile is prescribed at $x=0$, whereas the outlet is left free, i.e. the associated natural boundary condition is zero traction. The temperature is prescribed to $\vartheta=1$ at the bottom wall $y=0$ and to $\vartheta=0$ at the top wall $y=1$. The inlet and outlet are considered adiabatic.

This problem was solved in [19] as a benchmark for open boundary flows using a finite-difference method and a fine grid. It can be considered as a model for several relevant engineering problems, such as the fabrication of microelectronic circuits using the chemical vapour deposition process (cf. [19], see references therein).

The dimensionless parameters of the problem have been taken as $\operatorname{Re}=10, F r=1 / 150$, and $P e=40 / 9$ (the average inlet velocity, the height of the channel and the temperature difference between the top and bottom walls have been chosen as reference values for velocity, length, and temperature, respectively). These parameters are the same as in [19] except for the Péclet number, which is slightly higher in that work $(P e=20 / 3)$. In both cases, these values result in a thermoconvective instability of the basic Poiseuille flow. The linear stability analysis of unstable stratified plane Poiseuille flow in a infinite horizontal channel can be found in [20]. It is shown there that the form of the instability could vary from travelling transverse waves to longitudinal rolls, with axes parallel to the main flow direction and thus leading to a three-dimensional flow pattern. Travelling transverse waves are found for small values of the Rayleigh number. This is the situation for the dimensionless parameters used here and therefore a two-dimensional calculation is possible. It should be remarked, however, that three-dimensional effects are, in general, very important for thermally coupled flows [21].

The domain $[0,10] \times[0,1]$ has been discretized using two uniform meshes of $16 \times 40$ and $50 \times 100$ bilinear elements, respectively. For the length of the channel considered, it is concluded in [19] that the numerical solution is not affected by the artificial boundary conditions for $2 \leqslant x \leqslant 8$.

Some results of the calculation on the fine mesh are shown in Figures 1-3. They display the streamlines, temperature contours, and pressure contours obtained at two time steps (roughly) 


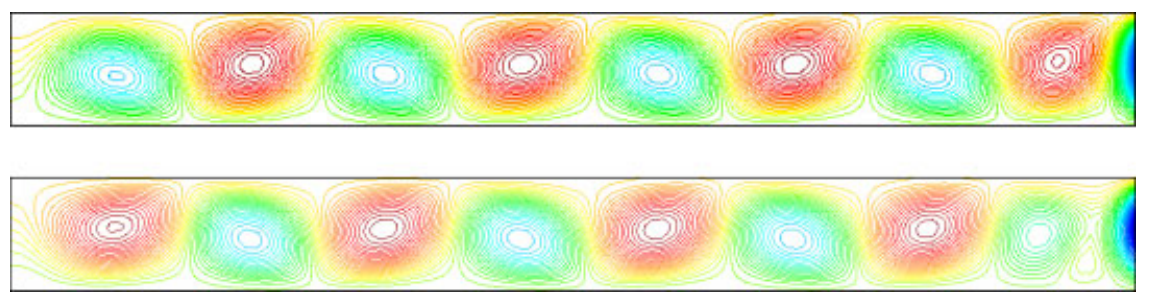

Figure 1. Streamlines at two different time steps for the plane Poiseuille flow example.
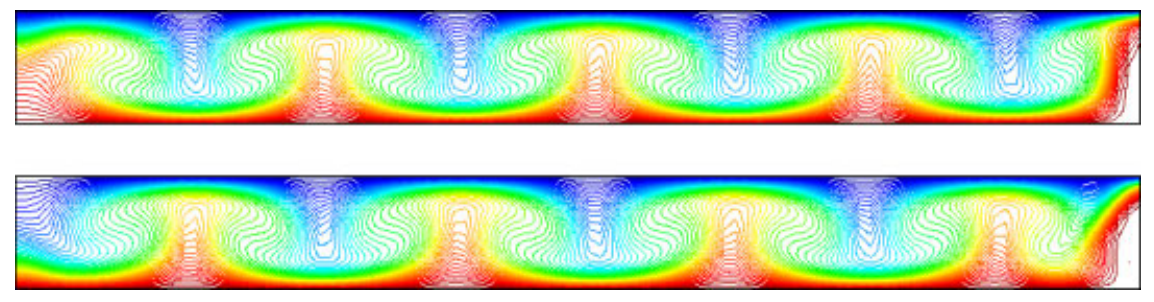

Figure 2. Temperature contours at two different time steps for the plane Poiseuille flow example.
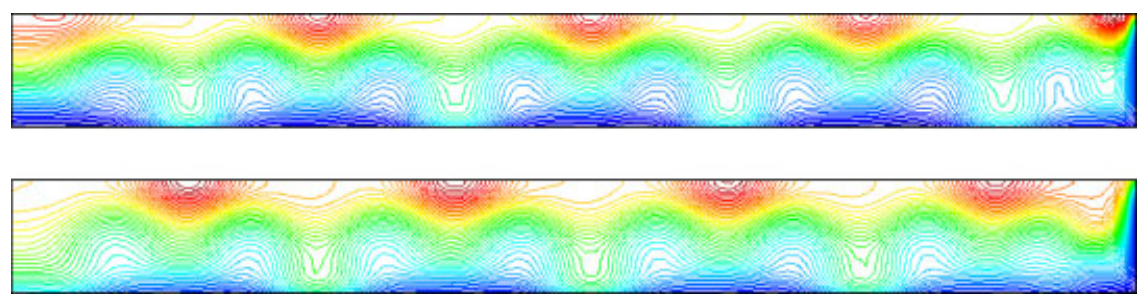

Figure 3. Pressure contours at two different time steps for the plane Poiseuille flow example.

half-a-period apart. The bad influence of the artificial boundary conditions can be observed, especially in what concerns the outlet wall. It is clear that the zero traction prescription does not reproduce the effect of an infinitely long channel. The proper evaluation of boundary conditions necessary for the numerical simulation of flows in infinite domains is an area that still deserves further research.

The important point is the comparison of the results obtained using QSS and DS. To do this, we compare the evolution in time of velocity and pressure at the central point of the computational domain, $(x, y)=(5,0.5)$. Results using $\delta t=0.02$ on the fine mesh and $\delta t=0.1$ on the coarse mesh are shown in Figure 4. For the DS case, two options have been considered, namely, a second-order time integration of the subscales, labelled DS2 in Figure 4, and a first-order time integration, labelled DS1. From Figure 4 the following observations can be made:

- Results using DS1 and DS2 are very similar. This confirms the discussion of Section 2.3 about the feasibility of using DS1 and keeping the order of approximation.

- DS2 has spurious high-frequency oscillations that are removed using DS1. This is to be expected, since it is known that the Crank-Nicolson scheme is unable to remove high frequencies. 

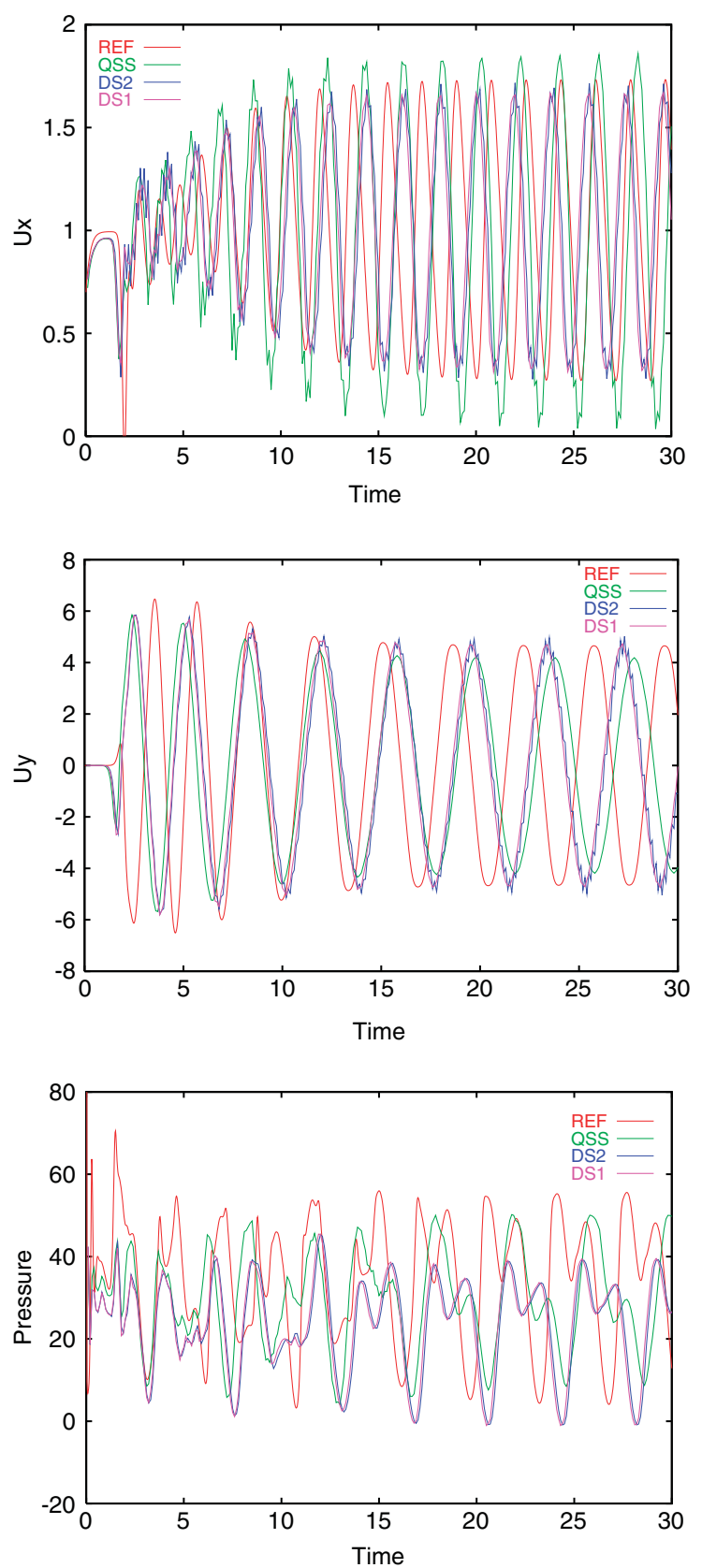

Figure 4. Time evolution at the central point for the plane Poiseuille flow example. Time step $\delta t=0.1$. Top: horizontal velocity; middle: vertical velocity; bottom: pressure. REF: reference solution; QSS: solution with quasi-static subscales; DS2: dynamic subscales with second-order time integration; DS1: dynamic subscales with first-order time integration. 


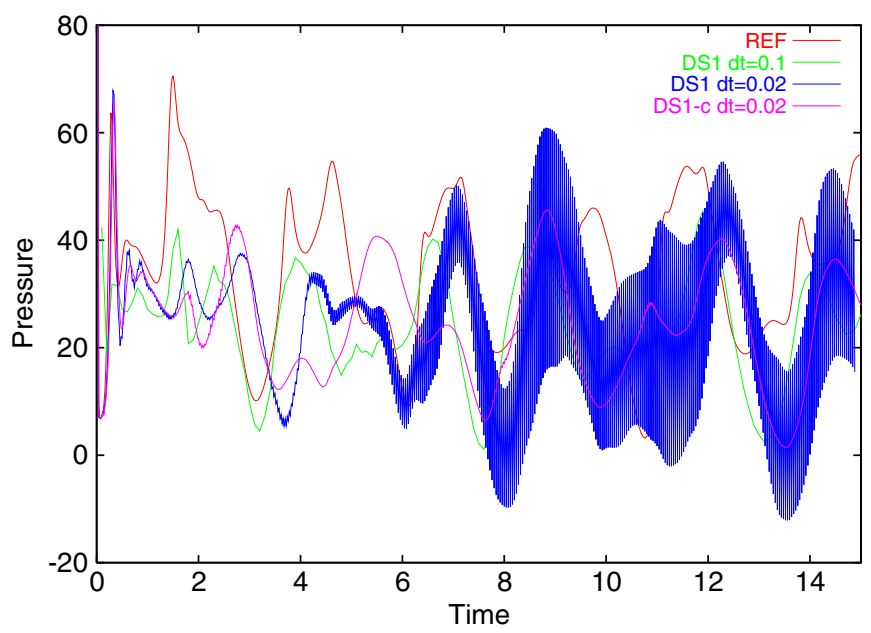

Figure 5. Pressure evolution in time at the central point for the plane Poiseuille flow. REF: reference solution; DS1: dynamic subscales with first-order time integration without $\delta_{t} R_{p}^{n}$ in the pressure subscale. DS1-c: same as DS1 but including $\delta_{t} R_{p}^{n}$ in the pressure subscale.

Our approximation to the subscales is non-smooth (they are discontinuous from element to element), and those high-frequency components will be probably present.

- DS results are much more accurate than QSS, since they are closer to the reference results (obtained using QSS on the fine mesh).

- QSS results have some spurious oscillations in velocity that do not appear using DS. This is an important fact, since QSS are the results obtained with what can be considered a standard stabilized finite-element method.

As a conclusion, results using DS1 seem to be excellent. Nevertheless, it is interesting to show in this example the effect of the term $\delta R_{p}^{n}$ in (23). When $\delta t=0.1$, this term is not important, but when $\delta t=0.02$ its omission leads to a very important pressure oscillation from time step to time step using DS1. Figure 5 shows this oscillation, together with the results obtained including $\delta R_{p}^{n}$ in (23), which are completely free of spurious oscillations.

\subsection{Transient natural convection of low-Prandtl-number fluids}

In this example, the transient convective motion of a fluid enclosed in a unit square cavity driven by a temperature gradient will be numerically analysed. The left vertical wall is suddenly heated and maintained at a constant temperature, while the right vertical wall is maintained at the initial temperature. Horizontal walls are assumed to be adiabatic, i.e. the zero heat flux boundary condition is prescribed. Homogeneous Dirichlet boundary conditions are prescribed everywhere on the boundary for the velocity.

The only dimensionless parameters involved in the problem are the Prandtl number $\operatorname{Pr}$ and the Rayleigh number $R a$ or, equivalently, the Grashof number $G r$. Numerical results will be presented for $\operatorname{Pr}=0.005$ and the value $G r=5 \times 10^{6}$.

The value $P r=0.005$ is very small and not often encountered in common fluids. For example, the Prandtl number is 0.71 for air, 7.03 for water, and 0.0249 for mercury (at $293 \mathrm{~K}$ ). Small values 

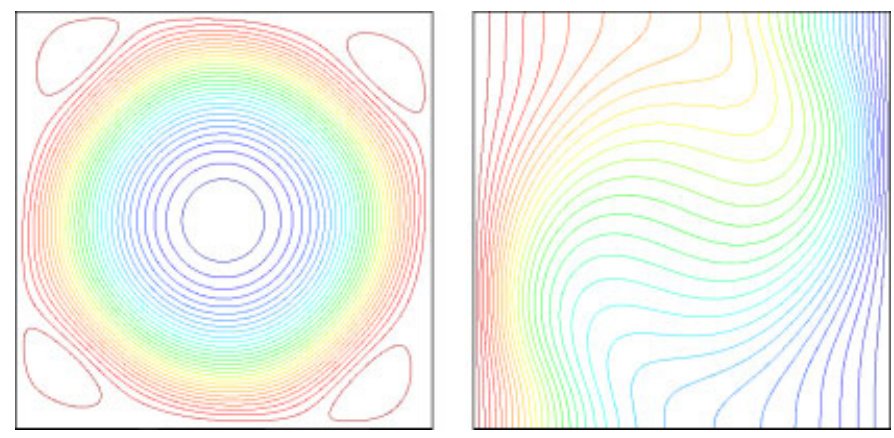

Figure 6. General streamline pattern (left) and temperature contours (right) for the flow in cavity at low Prandtl number.
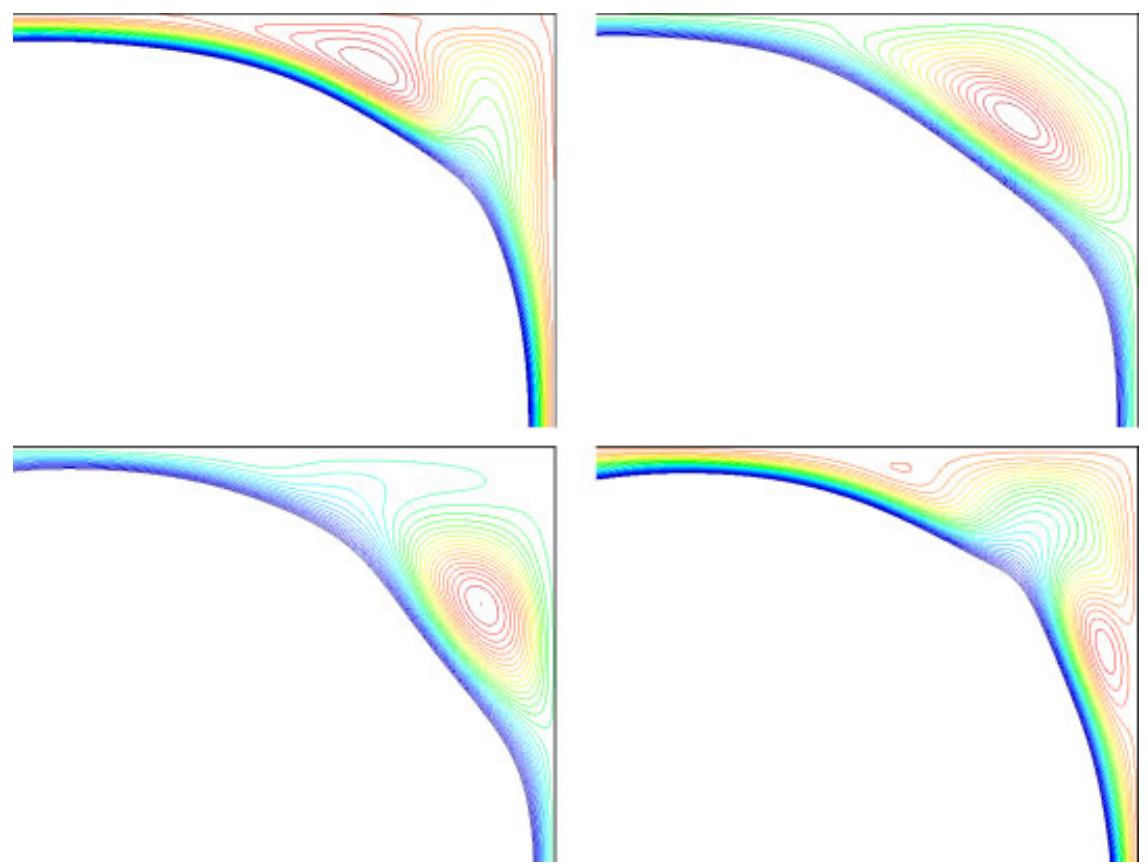

Figure 7. Evolution (from left to right and from top to bottom) of the streamlines at the top right corner of the cavity for the flow in cavity at low Prandtl number.

of $\mathrm{Pr}$ are typical of liquid metals and semiconductors. The problem to be studied now is relevant to the solidification of ingots and casting, crystal growth from melts, material processing, nuclear reactor safety, and other applications (cf. [22]).

Although the problem just described is a very popular test for thermally coupled flows when $\operatorname{Pr}$ is high, the interest for solving low-Prandtl-number flows is that this problem is not yet well understood. It is found that the flow exhibits a periodic oscillation when the Grashof number exceeds a critical value. In particular, for $\operatorname{Pr}=0.005$ a steady-state solution is obtained for $G r=3 \times 10^{6}$ 

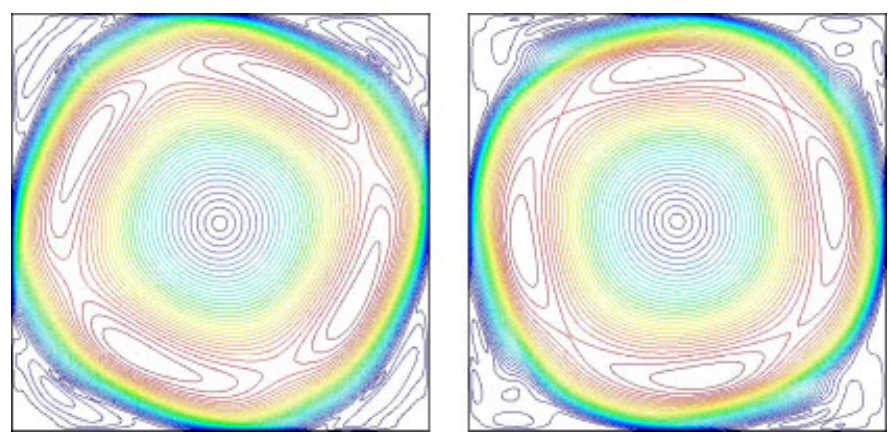

Figure 8 . Velocity norm at two different time steps separated half a period for the flow in cavity at low Prandtl number.
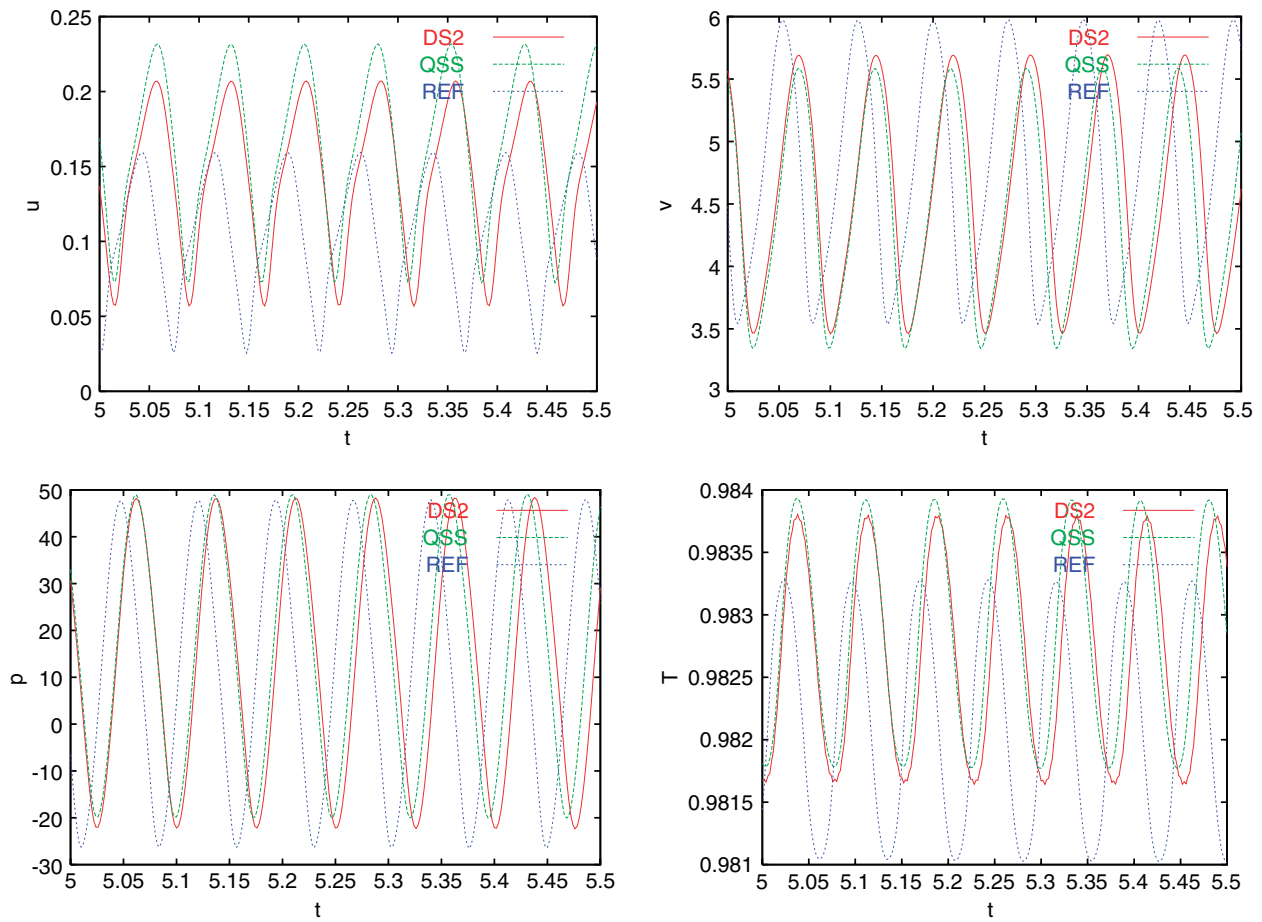

Figure 9. Comparison of results at point 1: $(x, y)=(0.006,0.5)$ for the flow in cavity at low Prandtl number. $u$ : horizontal velocity; $v$ : vertical velocity; $p$ : pressure; $T$ : temperature.

but the solution bifurcates and for $G r=5 \times 10^{6}$ an oscillatory flow field is found. For further information about this problem the reader is referred to [22], from where this problem has been taken. Our purpose here is to demonstrate the efficiency of the numerical method proposed in this work.

Two meshes of bilinear finite elements have been used in the calculations. The 'coarse' one is made of $60 \times 60$ elements, refined near the walls of the cavity. The 'fine' mesh is made of $180 \times 180$ 

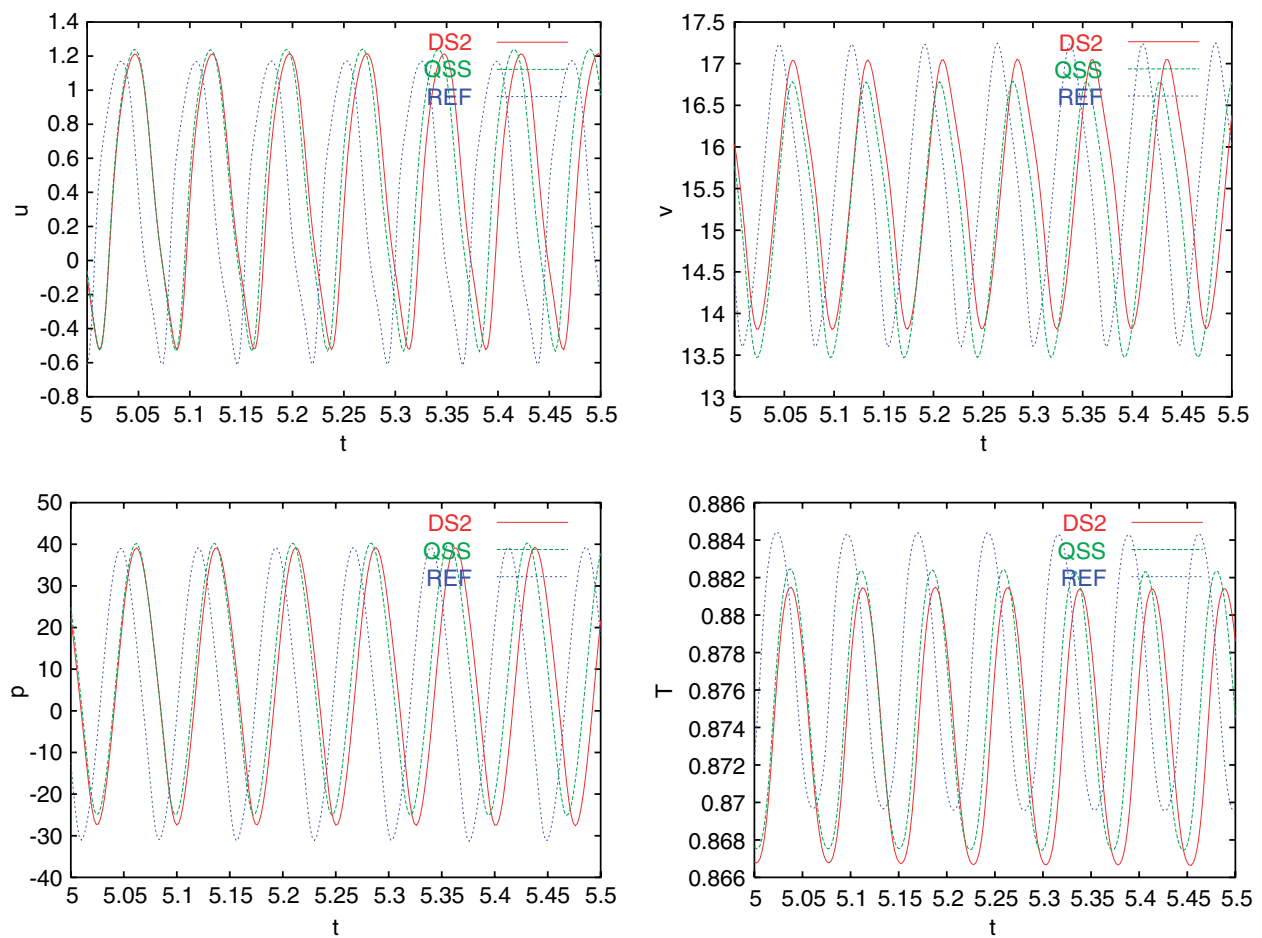

Figure 10. Comparison of results at point 2: $(x, y)=(0.0438,0.5)$ for the flow in cavity at low Prandtl number. $u$ : horizontal velocity; $v$ : vertical velocity; $p$ : pressure; $T$ : temperature.

elements, and it is also refined near the walls. The time step has been taken as $\delta t=0.002$ in both cases. A remark is needed concerning the consequence of this choice for the time integration of the Navier-Stokes and the temperature equations. The critical time step of the backward Euler scheme, obtained by taking $\beta=0$ in (19)-(21), is approximately $\tau_{1}$ for (19) and $\tau_{3}$ for (21) [6]. Due to the low Prandtl number of the flow, the temperature equation is dominated by thermal diffusivity, whereas convective effects are important only in the Navier-Stokes equations. It turns out that the ratio $\delta t / \tau_{1}$ is 4.77 for the coarse mesh and 15.35 for the fine one, indicating that $\delta t$ is comparable with $\tau_{1}$. However, the ratio $\delta t / \tau_{3}$ is 222 for the coarse mesh and 2000 for the fine one. Therefore, the time step $\delta t=0.002$ is very 'large' for the time integration of the temperature equation and, as a consequence, not much influence is to be expected between QSS and DS, particularly when diffusive effects dominate, as in boundary layers. Numerical results confirm this fact, as we shall show.

Let us discuss now the results of the numerical simulation. The general flow pattern is shown in Figure 6 at a time step when the flow is fully developed. It is observed that there is a main central vortex and also that vortices appear at each corner of the cavity. These small vortices move in clockwise sense, being created from flow detachment at the walls, growing and then collapsing against the walls. This evolution for the top right vortex can be observed in Figure 7 . It is seen how the vortex is originated from the top wall, moves in the clockwise sense while grows, and then decreases until it reaches the right wall. Before it completely disappears, a new vortex appears at the top wall. The contours of the velocity norm are plotted in Figure 8. These results correspond 

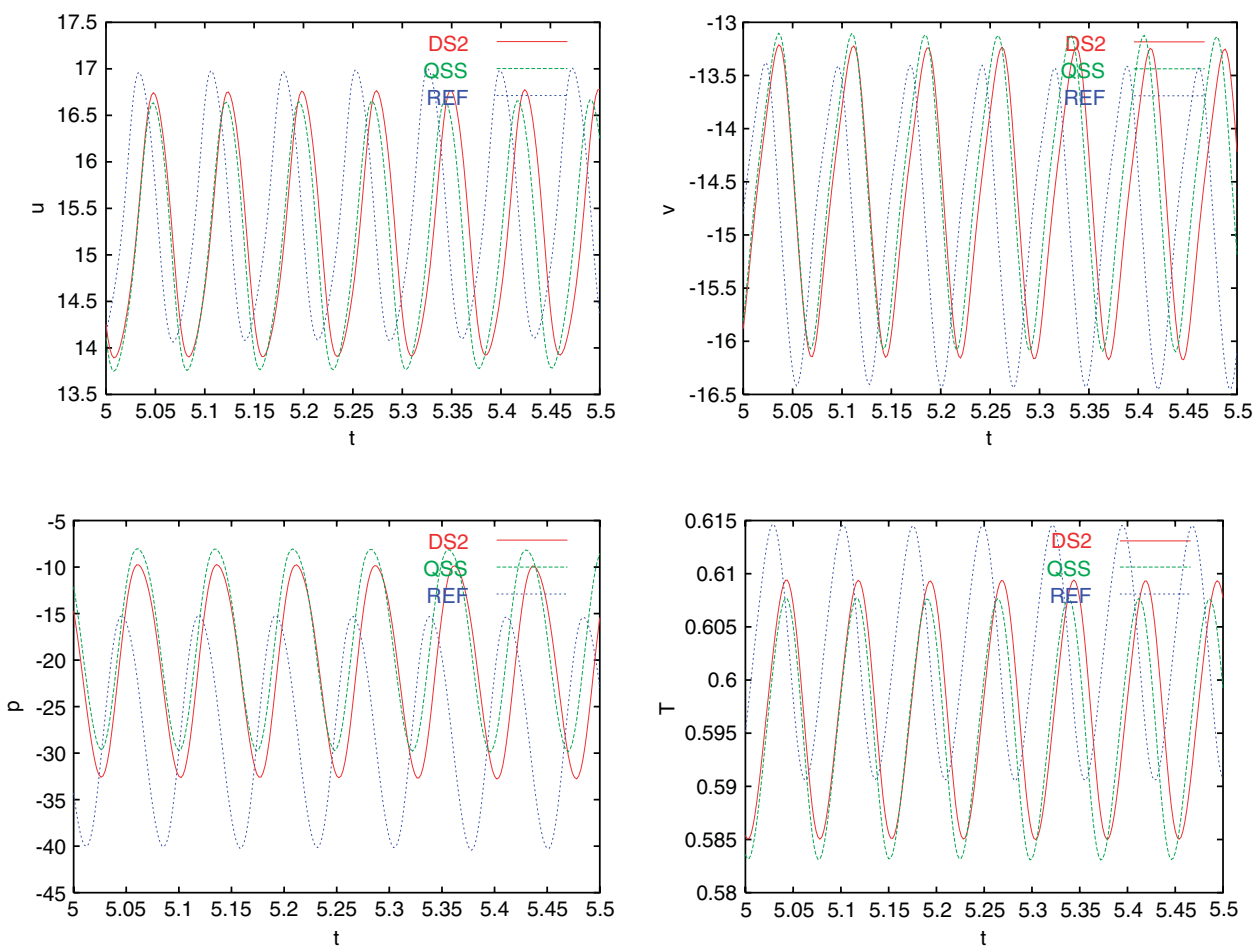

Figure 11. Comparison of results at point 3: $(x, y)=(0.773,0.773)$ for the flow in cavity at low Prandtl number. $u$ : horizontal velocity; $v$ : vertical velocity; $p$ : pressure; $T$ : temperature.

to time steps separated by half a period (approximately). They show that the main vortex pulsates, increasing and decreasing the flow magnitude periodically. All these results have been obtained with the fine mesh and QSS.

To compare the performance of QSS and DS we have considered three representative points. Point 1 is located at $(0.006,0.5)$, point 2 at $(0.0438,0.5)$, and point 3 at $(0.773,0.773)$. The first two points lie inside the boundary layer formed at the left wall, whereas the third one is placed at the top right position of the main vortex. Figures 9-11 show the evolution in time of the flow variables (horizontal velocity, vertical velocity, pressure, and temperature) at points 1, 2, and 3, respectively. From these pictures it is observed that all flow variables are more accurate using DS than QSS at points 1 and 3, whereas the results are inconclusive at point 2, where temperature seems to be slightly better using QSS (although the differences with DS are very small). The rest of flow variables are slightly better reproduced using DS. The explanation we give to this fact relies on the previous discussion about the size of the time step. As mentioned earlier, this time step is large for the heat equation, and thus QSS and DS should perform similarly, as it is observed in the numerical experiments. This is particularly so in boundary layers, since diffusive effects dominate there. At other sampling points of the computational domain, DS performs consistently better than QSS, in accordance with the results of the previous example. In this particular example, both the finite-element equations and the equations for the subscales have been integrated in time with second-order accuracy. 


\section{CONCLUSIONS}

The aim of this paper has been to explain how to deal with dynamic subscales in the finite-element approximation of thermally coupled flows using the Boussinesq approximation. The space variation of the subscales is approximated in terms of the residual of the finite-element unknowns, in the classical way used in stabilized finite-element methods, but now they are integrated in time.

From the conceptual point of view, the formulation presented has several benefits, inherited from the formulation applied to isothermal flows [2] (improved time stability and accuracy, particularly when $\delta t \rightarrow 0$, correct behaviour of the stabilization parameters with the time step size, commutation of space and time discretization). Additionally, in the case of thermally coupled flows the coupling of velocity and temperature subscales is dealt with in a natural way.

Several computational aspects of the formulation have been discussed, in particular, the way to deal with the nonlinear algebraic problem for the subscales that needs to be solved at each time step and two simplifications to decrease the storage needs of the formulation.

The results of the numerical experiments conducted confirm the conclusions drawn for isothermal flows and that make the formulation particularly appealing:

- The formulation is more accurate than considering the subscales quasi-static.

- Some oscillations encountered using QSS are removed.

The last item is especially significant when the subscales are integrated in time using a first-order scheme, which avoids high-frequency spurious oscillations in the tracking of the subscales in time.

\section{ACKNOWLEDGEMENTS}

This research has been supported by project VEM2003-20069-C03-03 of the Spanish Ministerio de Ciencia y Tecnología. J. Principe also acknowledges the support received from the Departament d'Universitats, Recerca i Societat de la Informació of the Generalitat de Catalunya (Catalan Government) and the European Social Fund through a doctoral grant.

\section{REFERENCES}

1. Hughes TJR, Feijóo GR, Mazzei L, Quincy JB. The variational multiscale method-a paradigm for computational mechanics. Computer Methods in Applied Mechanics and Engineering 1998; 166:3-24.

2. Codina R, Principe J, Guasch O, Badia S. Time dependent subscales in the stabilized finite element approximation of incompressible flow problems. Computer Methods in Applied Mechanics and Engineering, in press.

3. Baiocchi C, Brezzi F, Franca LP. Virtual bubbles and Galerkin/least-squares type methods (Ga.L.S). Computer Methods in Applied Mechanics and Engineering 1993; 105:125-141.

4. Codina R. Stabilized finite element approximation of transient incompressible flows using orthogonal subscales. Computer Methods in Applied Mechanics and Engineering 2002; 191:4295-4321.

5. Codina R. A stabilized finite element method for generalized stationary incompressible flows. Computer Methods in Applied Mechanics and Engineering 2001; 190:2681-2706.

6. Codina R, Zienkiewicz OC. CBS versus GLS stabilization of the incompressible Navier-Stokes equations and the role of the time step as stabilization parameter. Communications in Numerical Methods in Engineering 2002; 18:99-112.

7. Shakib F, Hughes TJR. A new finite element formulation for computational fluid dynamics: IX. Fourier analysis of space-time Galerkin/least-squares algorithms. Computer Methods in Applied Mechanics and Engineering 1991; 87:35-58.

8. Tezduyar T, Sathe S. Stabilization parameters in SUPG and PSPG formulations. Journal of Computational and Applied Mechanics 2003; 4:71-88. 
9. Bochev PB, Gunzburger MD, Lehoucq RB. On stabilized finite element methods for the Stokes problem in the small time-step limit. International Journal for Numerical Methods in Fluids 2007; 53:573-597.

10. Badia S, Codina R. On a multiscale approach to the transient Stokes problem. Transient subscales and anisotropic space-time discretization, submitted for publication.

11. Hughes TJR, Wells GN. Conservation properties for the Galerkin and stabilised forms of the advection-diffusion and incompressible Navier-Stokes equations. Computer Methods in Applied Mechanics and Engineering 2005; 194:1141-1159.

12. Gibbon J, Titi E. Attractor dimension and small length scale estimates for the three dimensional Navier-Stokes equations. Nonlinearity 1997; 10:109-119.

13. Calo VM. Residual based multiscale turbulence modeling: finite volume simulations of bypass transition. Ph.D. Thesis, Department of Civil and Environmental Engineering, Stanford University, Palo Alto, CA, 2004.

14. Hoffman J, Johnson C. A new approach to computational turbulence modeling. Computer Methods in Applied Mechanics and Engineering 2006; 195:2865-2880.

15. de Sampaio PAB, Hallak PH, Coutinho ALGA, Pfeil MS. A stabilized finite element procedure for turbulent fluid-structure interaction using adaptive time-space refinement. International Journal for Numerical Methods in Fluids 2004; 44:673-693.

16. Gravemeier V. The variational multiscale method for laminar and turbulent flow. Archives of Computational Mechanics-State of the Art Reviews 2006; 13:249-324.

17. Hughes TJR. The Finite Element Method. Prentice-Hall: Englewood Cliffs, NJ, 1987.

18. Codina R. A nodal-based implementation of a stabilized finite element method for incompressible flow problems. International Journal for Numerical Methods in Fluids 2000; 33:737-766.

19. Evans G, Paolucci S. The thermoconvective instability of plane Poiseuille flow heated from below: a proposed benchmark solution for open boundary flows. International Journal for Numerical Methods in Fluids 1990; 11:1001-1013.

20. Gage KS, Reid WH. The stability of thermally stratified plane Poiseuille flow. Journal of Fluid Mechanics 1968; 33:21-32.

21. Kessler R. Nonlinear transition in three-dimensional convection. Journal of Fluid Mechanics 1987; 174:359-379.

22. Mohamad AA, Viskanta R. Transient natural convection of low-Prandtl-number fluids in a differentially heated cavity. International Journal for Numerical Methods in Fluids 1991; 13:61-81. 